\title{
Los «Bienes Democráticos» en la Economía del Bien Común: cuatro vías para su delimitación conceptual
}

\author{
Luis F. Campos Saavedra \\ Carmen Rodríguez Morilla
}

RESUMEN: ¿Es posible delimitar una nueva categoría económica -los Bienes Democráticos (BBDD)- para poner en marcha y operativizar un nuevo marco dentro del cual concebir la gestión de bienes más indispensables como derechos económicos, por su papel central en la realización de los Derechos Humanos? ¿De qué manera podríamos identificar tales Bienes Democráticos? ¿Qué directrices o principios rectores guiarían su búsqueda? Para responder a estas preguntas presentamos una propuesta con la que tratamos de proporcionar los fundamentos necesarios para establecer su delimitación conceptual, desde cuatro aristas o vías que le dan contención: (1) La sociohistórica, con un recorrido por las grandes transformaciones socioculturales de la Humanidad, en las que los BBDD se ponen de manifiesto, con un carácter vital y fundacional para la condición humana. (2) La económica: con la articulación de los BBDD dentro del amplio campo de los commons, los elementos diferenciales que los distinguen, la cuestión de la reciprocidad sistémica y los tramos, en los que cada BBDD se especifica y concreta. (3) La política: que comienza con la toma de conciencia de la escasa institucionalización y funcionalidad real de las comunidades -el sujeto activo que ha de gestionar estos bienes-, que al organizarse amplía el espacio que define las posibilidades políticas, creando gobernanza. (4) La jurídica: que pone en relación el modo de gestión de los bienes económicos señalados con algunos de los derechos fundamentales, estableciendo derechos económicos. Galtung (1977), Bruni y Zamagni (2007) y Felber (2010) nos aportan los puntos de partida.

PALABRAS CLAVE: Bienes comunes urbanos, bienes democráticos, derechos económicos, necesidades humanas básicas.

CLAVES ECONLIT: 035, P32, P46, P47, P48.

Cómo citar este artículo / How to cite this article: CAMPOS SAAVEDRA, L.F. \& RODRÍGUEZ MORILLA, C. (2017): "Los «Bienes Democráticos» en la Economía del Bien Común: cuatro vías para su delimitación conceptual", CIRIEC-España, Revista de Economía Pública, Social y Cooperativa, 90, $223-252$.

Correspondencia: Luis F. Campos Saavedra, doctorando de la Universidad de Sevilla, Oluife@ gmail.com; y Carmen Rodríguez Morilla, Profesora de Economía Aplicada II de la Universidad de Sevilla, cmorilla@us.es. 


\section{EXPANDED ABSTRACT}

\section{«Democratic Goods» in the Economy for the Common Good: four ways for conceptual delimitation}

\section{OBJECTIVE}

Through this work, with an introductory intention, we will try to find a line of argument that will serve to delimit conceptually a new economic category that specifically accounts for the ideal management mode for the most indispensable assets as economic rights, within the large type of property known as commons or communal goods. How could we identify such goods? Which guidelines or guiding principles would guide our search? With what terms could we refer to the new category?

\section{APPROACH}

The Economy for the Common Good (ECG) was formulated by Felber in 2010. We will develop our proposal within this general theoretical/reflexive framework. After synthesizing their core lines, we analyze their possible repercussions as a model of political economy and pay attention to their role as a vector for domesticating, eroding, and finally transcending the capitalist ways and practices and the serious imbalances it causes. We start from Communities for the Common Good, as civil society entities, that manage a small set of assets in community that assures them resilience: guaranteeing more autonomy, self-sufficiency and own initiative; at the same time as less dependence on other instances like market or State, with their unsecured wages or their subsidies. Resilience truly represents and constitutes the helm in advancing our purpose: communities for the common good must be unequivocally resilient communities.

Common sense quickly gives us the list of indispensable goods, of which we daily face a massive and continuous consumption: housing, food, clothing, mobility and electric and internet access bills. We leave aside common sense pre-notions to proceed, as Bourdieu explains, to the epistemological break with which to be able to achieve them in a more rigorous and scientific way. We propose to approach this rupture through four ways:

1. The sociohistorical way involves a journey through the Humanity's greatest socio-cultural transformations. It is a History of Great Changes within the evolution of human culture, paying attention to the goods that have had a vital and foundational character during the journey, marking each of the moments of transformation (discontinuities) between each historical or cultural continuity and its immediately subsequent. There are four discontinuities we identify as fundamental: 


\section{LOS “BIENES DEMOCRÁTICOS» EN LA ECONOMÍA DEL BIEN COMÚN: CUATRO VÍAS PARA SU DELIMITACIÓN CONCEPTUAL}

a) the passage from Paleolithic to Neolithic, with the discovery of agriculture that made possible the first settlements, which we update operationally as homes or housing.

b) the transition from Neolithic to complex societies (Mesopotamia, Nile Valley, Indus Valley) characterized by the incorporation of irrigation systems that multiplied agricultural production, forcing the need for a graphic system for accounting, which will later become more complex as a writing system.

c) the passage from complex societies to industrial society. The large quantity and density of energy that allowed the massive and systematic use of fossil fuels and their ability to vectorize themselves to promote mobility play the same role as agriculture, housing and water distribution had played in the two previous discontinuities.

d) the transition from industrial society to network society. A leap that is marked by the emergence of internet with all its potential in terms of massive intercommunication between peers in the network, revolutionizing the economic modes of production (3D printing) distribution (peer-topeer) and consumption (collaborative economy).

The goods that have functioned as triggers of these great transformations, properly establishing themselves as the material conditions of possibility of these human realizations, currently provide the material conditions of human subsistence and dignity according to the civilizing paradigm that is taken into account as life context. On them lies resilience of civil society as such; when the communities of which it is composed are empowered with the control, leadership and management of these resources. Putting together all the stages studied we obtain six sectors, which for all above can be called foundational goods: housing, agroecology, water, energy, mobility and the internet.

2. The economic path is another channel towards the new category that we aim to achieve. The broad field of commons -communal assets from ECG's perspective- is already consolidated after Elinor Ostrom's laureate works. We recognize its importance in setting up a format for community ownership. Civil economy and basic needs economy are theories that complete the essential axes to concretize the premises in order to justify the incorporation of civil society in the framework of the economic management modes. They can be called community goods: the asets to be managed through this type of community-based property.

3. The political path begins with the awareness of the scarce institutionalization and functionality of civil society, to which is added the hitherto unknown knowledge of the role of communities in the whole of an Economy for the Common Good, as the subject on whom all possibility and responsibility for social or community resilience lies. When civil society organizes itself for the management of these goods, it elevates itself as the third pillar -along with the market and the State- of social order, expanding the space of political possibilities as it opens the door to governance relations. The main character to be enshrined by the new mode of management for the goods on which human dignity and subsistence depend, and hence its community resilience, is democracy. Now, from this perspective, we can call them democratic goods. 
4. The juridical way aims first to raise awareness of the relationship between the list of assets obtained by the development of previous channels of analysis and the realization of human rights, thus pointing to the establishment of economic rights. Secondly, he stresses the importance of extending to the economic order the division principle that separates the fundamental right from the ordinary one in the legal order. In this way, the goods grouped in the previous lines of reasoning could be called as fundamental goods, or rather, by the legal place that would correspond to them, constituent goods.

\section{RESULTS / IMPLICATIONS}

Of the four possibilities that we have obtained for its denomination, namely: foundational, communitarian, democratic and constituent, the attribute of "democratic" is the only one that is in permanent revision. At any historical moment, the modes of practical management of asets may undergo changes that enable or improve, for example, collective intelligence flow, due to advances in technology. In that case, the format used for its management should be updated so that it always coincides with the one that offers the highest levels of codecision, democracy and transparency of its time. This need for constant updating is the reason why we are finally inclined to preferentially denominate them as democratic goods, although the other three denominations are perfectly compatible and acceptable.

To facilitate the understanding of the concept, we offer a first formulation of its definition, along with some other important features. Each of Galtung's basic needs -survival, welfare, freedom and identity- suggests a paired function among the goods we have found. The last step (identity) allows the incorporation of health and education, closing the complete scheme of democratic goods with eight sectors, as shown at the end, on the last page.

\section{PRACTICAL CONCLUSIONS AND ORIGINAL VALUE}

The Economy for the Common Good contains two nuclei for practical work -communal goods and communities- that allow us to progress substantially towards a new concept for the establishment of economic rights as part of universal rights. Although Felber's manual does not initially envisage a specific structure or function of its own for the communities, nor does it specify a certain format for the management of communal assets, those are, clearly, the active subject that is to manage the minimum package of indispensable goods, which we have called democratic goods. Communal goods provide the type of general management -with a marked non-specific character, as Ostrom argued- that is viable for a community. It is through the most democratic mechanisms, such as self-consumption and cooperation structures oriented to basic needs -democratic goods cooperatives, very similar in their operations to consumers cooperatives- how communities gain resilience.

The substitution of oligopolies by self-organized communities in the most indispensable sectors also offers a landscape of remarkable reduction of the total carbon footprint of our civilization. In the calculation of the economy's decarbonization both direct and indirect effects should be added. 


\section{LOS “BIENES DEMOCRÁTICOS» EN LA ECONOMÍA DEL BIEN COMÚN: CUATRO VÍAS PARA SU DELIMITACIÓN CONCEPTUAL}

The political and economic repercussions of democratic goods, managed as such, are not restricted to the environment, but rather enter into the already abundant discussion of the inclusion of universal basic income in our economic systems. A kind of management like the one proposed with this new concept immerses us in the possibilities of a basic income in resources, without prejudice to a possible complementation in currency, also softening some of its classic points of friction.

KEYWORDS: Basic human needs, democratic goods, economic rights, urban commons.

\section{1.- Introducción: la Economía del Bien Común como modelo}

A pesar de las dificultades,

a pesar de las señales de horror de nuestra época, el paraíso está aquí, tan próximo, que nos cuesta percibirlo

Rolando Toro

De manera equilibrada, en su más reciente artículo, Erik Olin Wright (2015:1) reconoce al capitalismo de nuestras sociedades ciertos elementos de fuerza, como el aumento de la productividad, la innovación tecnológica o la amplia difusión de bienes de consumo, al tiempo que enumera los copiosos daños que ocasiona, entre ellos, la destrucción de los medios de vida para una gran cantidad de personas, la precariedad para la zona inferior del mercado de trabajo o los tediosos y alienantes empleos que ofrece para la mayoría, perpetuando formas innecesarias de sufrimiento humano, por no hablar de la masiva destrucción que ocasiona al medioambiente. Concepciones como crecimiento ilimitado o sociedad de consumo que anteriormente habían sido asumidos como presupuestos casi incuestionables, hoy se consideran mitos. Y se adentra de inmediato en la búsqueda de tipos alternativos de economía, descubriéndonos cuatro grandes tendencias, con potencial para encaminarnos hacia la superación del modelo capitalista:

a) Aplastarlo, como pretende el socialismo revolucionario, que aprovecha el poder del Estado para destruirlo, tomando a su cargo una planificación para la economía.

b) Domesticarlo, como ha ensayado la socialdemocracia en Europa, tratando de compensar con impuestos sus graves desequilibrios.

c) Evitarlo, escapando de él, como practican quienes se recluyen en micro-sociedades contraculturales o marginales. 
d) Erosionarlo, a través de fórmulas integradas en economías de mercado, no puramente capitalistas, que replantean la actividad económica desde las relaciones sociales, el peer to peer (entre iguales), sin fines de lucro, en redes desnucleadas de consumo colaborativo o en cooperativas de consumidores, productores o trabajadores, y algunas otras posibilidades.

Es esta última disposición la que evidencia ciertos destellos para la pretensión de trascender las estructuras e instituciones capitalistas desde la esfera micro-social, la que abre puertas para quienes toman la acción de emprender y consumir en cualquier lugar por entre los estratos sociales -también los de más abajo- sumando para un cambio de paradigma económico, con alternativas capaces de ejercer erosión eficaz sobre el propio sistema, como reconoce Wright. Y nos recuerda que, una forma de desafiar al capitalismo consiste en "la construcción de unas relaciones económicas más democráticas, igualitarias y participativas en los espacios y grietas dentro de este complejo sistema allá donde sea posible [para a continuación determinarse por] ampliar y defender esos espacios", (Wright 2015:11) con la idea de desplazarlo, del modo en que una especie exógena puede encontrar su espacio dentro de un ecosistema y prosperar y desplazar a las especies endémicas dominantes, transformando el carácter del ecosistema en su conjunto. De esta misma forma fue como, durante un largo y sinuoso proceso de varios siglos, la sociedad feudal fue poco a poco erosionada por las estructuras proto-capitalistas antes de pasar a una posición dominante durante los dos últimos siglos.

No obstante quisiera reconocer, también con Wright, que la domesticación del capitalismo que pareciera equívocamente haber quedado fuera de nuestra mirada, juega un papel importante, como contraparte que complementa lo descrito hasta ahora. Erosionar la economía capitalista desde las instituciones de abajo o microeconómicas, con formas no capitalistas de actividades y relaciones económicas, "sólo es plausible si se combina con la acción de domesticarlo" (Wright 2015:13), desde las instituciones de arriba, macroeconómicas y legislativas. La imagen suscitada sería un proceso bilateral recíproco, dialéctico por cuanto reconoce que necesitamos domesticar al capitalismo de manera que se haga más erosionable -algo factible desde la participación en los movimientos políticos-, mientras lo erosionamos de manera que se vuelva más domesticable, para lo cual contaremos con proyectos que expandan formas emancipatorias de actividad económica. Diferimos del autor en que él despacha sin someter a cuestión la socialdemocracia como el locus del espectro de la economía política destinado a la domesticación de la economía capitalista, sin que esta asunción parezca crearle conflicto. Nosotros creemos, sin embargo, que este lugar está reservado para un nuevo modelo de economía que se abre paso reclamando un espacio propio, la Economía del Bien Común, "EBC" en lo sucesivo, que más abajo explicaremos. 


\section{LOS “BIENES DEMOCRÁTICOS» EN LA ECONOMÍA DEL BIEN COMÚN: CUATRO VÍAS PARA SU DELIMITACIÓN CONCEPTUAL}

\section{La Economía del Bien Común como economía política}

Debemos detenernos aquí para clarificar las razones de nuestra apuesta por la EBC como sistema que enmarca, con sus propios rasgos, una nueva economía política alternativa a las sucesivas adaptaciones que la corriente socialdemócrata ha sufrido en las últimas décadas. Cuando hablamos de política económica, "política" es sinónimo de "decisiones", pero cuando hablamos de economía política, la misma palabra torna su significado y pasa a ser sinónima de "Estado": la economía política establece el marco que regula el tipo de relaciones entre las instituciones del Estado y las instituciones del mercado. "Política económica" posee particularmente todo sujeto económico, tanto personas físicas como jurídicas y puede modificarse en cualquier momento. Por el contrario, la economía política sólo posee escasas opciones tipo y es determinada con anterioridad al juego de posibilidades que ella misma delimita o en los momentos de viraje político real de los gobiernos, en cuanto a economía se refiere. Las constituciones, ejemplo del primer caso, pueden establecer límites para definir entre qué extremos puede fluctuar la economía del país, siendo un hecho destacado que la Unión Europea ha establecido desde sus comienzos una economía de mercado en funcionamiento como requisito previo para cualquier posible adhesión de estados candidatos, dentro de los llamados "criterios de Copenhague"1.

Si bien la crisis de finales de los 80 en los países con economía planificada supuso el desprestigio de las políticas comunistas, las crisis de los años 30 y de 2008, con el crack bursátil y financiero, han dado al traste con cualquier esperanza acerca de las posibilidades de futuro de las políticas neoliberales. Explicar la EBC como modelo de economía política enfocando las diferencias con los modelos clásicos de países comunistas y capitalistas resultaría evidentemente más sencillo, pero menos revelador. En lugar de escoger esa opción, vamos a estrechar los márgenes a uno y otro lado para tratar de encontrar las distancias entre el modelo socialdemócrata, que Wright no se cuestiona, y la EBC, por ser ambos más cercanos en propósito y objetivos y también como modelos propiciatorios de domesticación del capitalismo.

De la economía socialdemócrata podemos asumir que se propone moldear y transformar los intereses del capital y del Estado en un paquete equilibrado entre la economía de mercado y el Estado de Bienestar (Held 2006) mientras que en la EBC "la búsqueda de beneficio y la competencia se transfiguran en esfuerzo hacia el bien común y la cooperación" (Felber 2010:260) con las debidas recompensas fiscales. Para ello, la aportación que cada parte realiza al bienestar de toda la sociedad en su conjunto se objetiva en un Balance del Bien Común que desplaza al Balance Financiero en empresas, con un nuevo propósito: prestar la aportación más grande posible al bienestar (2010:21), a lo que se añade un Producto del Bien Común como indicador macro que sustituye al PIB. A estas medidas en el ámbito empresarial se unen algunas limitaciones genéricas que redireccionan la economía hacia

1.- Las condiciones y principios que debe cumplir todo país que desee adherirse a la UE quedaron primeramente establecidas en los artículos 49 y 6 (apartado 1) respectivamente del Tratado de la Unión Europea. El Consejo Europeo de Copenhague de 1993 consolidó los requisitos, que finalmente fueron intensificados en el Consejo Europeo de Madrid de 1995. 
un sistema de retroalimentación negativa (2010:112), es decir, re-equilibrante, que empuja a la mayor parte de la población hacia la zona central de la distribución normal de las estadísticas socio-económicas. Límites en salarios, rentas y herencia. Instituciones nuevas, como una banca democrática. La democratización de la propiedad de los bienes en las grandes empresas hace por primera vez acto de presencia en una economía de mercado. Todo ello presidido por la solidaridad y la justicia social, la sostenibilidad ecológica, la participación democrática, la transparencia y la dignidad como altos valores. Felber reconoce que el modelo combina elementos de todos los sistemas económicos (2010:253), superando así la dicotomía abierta entre las propuestas liberales, de planificación económica y también otras, para maximizar el bien común. Todo ello tomando impulso a partir de una espiral de transformaciones desde todos los ángulos, lugares y sectores económicos con los cuales "la democracia experimentará un empuje vital" (Felber 2010:234). En cambio, la economía socialdemócrata opta por "dejan hacer" al capital con la esperanza de que el sistema de impuestos tendrá posteriormente capacidad de re-equilibrio fiscal. Veamos comparativamente objetivos y características en ambos sistemas para apreciar más diferencias.

Comparemos el esquema general de retroalimentaciones negativas, característico y definitorio de la EBC, que redirige continuamente al conjunto económico hacia el auto-equilibrio. La socialdemocracia no presenta grandes diferencias respecto del neoliberalismo, a la vista de la polarización socioeconómica, que se mantiene en las épocas en las que estos gobiernos se hacen con el poder. Por otro lado, en la socialdemocracia, las empresas mantienen la presión que el liberalismo ejerce sobre ellas para crecer en un continuo sin fin y aumentar su tamaño, mientras que en la EBC las empresas se ven liberadas de tales obligaciones y únicamente crece la aportación al bien de tod @s (Felber 2010:81,256). Veamos también la diferencia en cuanto a los sectores predilectos a los que se dirige el discurso. Mientras que la socialdemocracia se identifica con los trabajadores, a quienes pretende mejorar sus condiciones de vida mediante el refuerzo en las negociaciones laborales, la EBC toma no ya al ser humano sino a la vida misma en el centro de su acción (biocentrismo) al priorizar el medio ambiente, la dignidad y la felicidad vital de todas las personas sin distinción. La propuesta socialdemócrata aporta medidas de compensación, fundamentalmente a través de impuestos progresivos, para tratar de paliar las desigualdades, mientras que en la EBC los impuestos no poseen un papel central, porque las limitaciones de salarios, rentas, dotes de herencia y otras no dan pábulo a desequilibrios económicos tan acuciantes. Es decir, que mientras en el primero de los sistemas comparados la aportación principal es a posteriori, actuando de manera compensatoria o redistributiva, en el segundo las principales medidas tienen lugar a priori o mejor aún por efecto de las propias actividades económicas per se, distribuyendo proactivamente. En lo que respecta a la relación entre medios y fines, en el primer sistema, el fin no aparece prefigurado en los medios; en el segundo sí, aportando mayor coherencia, rasgo en el que encallan los socialdemócratas, algo que se recoge cuando se pregunta a la opinión pública. En definitiva, la socialdemocracia, que se propone domesticar al capitalismo, lejos de conseguirlo termina por consolidarlo como social-liberalismo. En cambio, la EBC, como conjunto de propuestas transformadoras sí parece albergar una capacidad real para el establecimiento de un escenario donde iniciativas emancipadoras puedan tener cabida y desarrollarse, e incluso ocupar una posición preeminente. Por último, nos ha faltado analizar la relación que ambos modelos tie- 


\section{LOS “BIENES DEMOCRÁTICOS» EN LA ECONOMÍA DEL BIEN COMÚN: CUATRO VÍAS PARA SU DELIMITACIÓN CONCEPTUAL}

nen con los derechos humanos (en adelante, DDHH), los ámbitos o esferas del orden social que contemplan en sus planteamientos y las relaciones sistémicas que estos modelos promueven entre ellas. Cuestiones que dejamos pendientes para abordar más adelante, cuando nos adentremos ya de lleno y con todo detenimiento en el objeto central de esta indagación científica: los bienes democráticos.

En la EBC reconocemos, consecuentemente, el modelo idóneo de cuantos están disponibles para la función esencial de domesticar el capitalismo con la que comenzábamos. Hay que resaltar que, a diferencia de los sistemas económicos anteriores a la EBC, a saber, neoliberalismo, socialismo revolucionario y propuestas intermedias como socialdemocracia o keynesianismo, la EBC proporciona un sistema económico capaz de provocar las dos funciones descritas a la vez. Queremos decir con ello que además de ejercer un efecto de domesticación desde la esfera estatal, en la medida en que la EBC es llevada a cabo e impulsada por empresas y agrupaciones territoriales no sólo de empresas - los llamados Campos de Energía- los agentes que la impulsan, identificados con el proceso, causan erosión de facto con sus propuestas, con su construcción del modelo en permanente y democrático perfeccionamiento y particularmente, con la transformación de sus entidades en entidades del Bien Común o con la puesta en marcha de otras de nuevo cuño, ante la perspectiva de que en tal caso la orientación al buen vivir pueda resultar aún mayor. Esta es la ventaja de un modelo que por vez primera no está pensado para ser aplicado una vez alcanzado el poder en la alta política, sino que su implantación es absolutamente plausible desde abajo, a través de:

a) actos de consumo consciente por parte de usuarios o consumidores,

b) el cambio de proveedores 0 de materia prima o de los modos de transformación productiva 0 del aumento de la cooperación entre iguales, por parte de las empresas,

c) la determinación política de valorar y promover de manera real los esfuerzos de las entidades anteriores y sumarse al proceso general, por parte de pequeñas corporaciones locales o ayuntamientos,

d) y también, como propondremos después, de asumir responsablemente la gestión de los bienes más indispensables, por parte de las comunidades.

Esta acción combinada, que podemos llamar de erosión-domesticación, probablemente posea efectos multiplicadores que en cualquier caso no tardaremos en observar, que Felber visibiliza cuando expresa cierta intención estratégica: la de "que crezca dentro de la economía de mercado un grupo que ya participa [de la EBC] -la zona del bien común-, mientras que aquellos que se resisten y se mantienen al margen, tengan que dar cada vez más explicaciones" (2010:229).

En este sentido, la EBC impulsa una descentralización de las soluciones porque todas las entidades: empresas, comunidades, administraciones, aportan su grano de arena para la solución de los pequeños o grandes problemas de la economía de la nación. Sucede así, con toda lógica, porque la EBC es un modelo con una metodología de implantación bottom-up (de abajo arriba), que permite una estrecha vinculación, incluso de identificación, de las partes integrantes con el propio sistema, a través de la puesta en acción de aquellas prácticas, que precisamente por ser éticas aumentan la 
satisfacción personal de estar obrando el cambio, un gran cambio al tiempo que se crean redes, como los Campos de Energía, en las que su acción es promovida, facilitada, reforzada y celebrada. Un modelo que ha nacido, no casualmente en pleno auge de la sociedad-red que pronosticaba Castells sino en viva correspondencia con ella. Nos disponemos ahora a recorrer los elementos principales que comprende el establecimiento de una economía del bien común.

\section{La Economía del Bien Común en acción}

En la economía real actual, el éxito económico se mide con indicadores puramente monetarios: el Balance Financiero para las empresas y el Producto Interior Bruto para el conjunto del país. Pero el primero no nos dice nada de si ese éxito lo están pagando niños que viven como esclavos o por el contrario disfrutan como escolares, si se produce a costa de evadir impuestos a paraísos fiscales o se contribuye al sostenimiento de las infraestructuras, si se causan daños al medio ambiente o se protege como condición para una vida saludable, si sus trabajadores están sumidos en la pobreza o alcanzan un nivel de vida aceptable, si se discrimina a las mujeres o se procura la simetría de género. El segundo, no nos dice nada acerca de si la ciudadanía atraviesa una guerra o dispone de un marco para la transformación pacífica de conflictos, si vive en una dictadura o en una democracia directa y de participación, si el consumo de recursos naturales agotables crece 0 se reduce, si siente miedo 0 confianza. En definitiva, no nos permite medir la felicidad de las personas, que es el resumen de lo que realmente nos importa, como seres humanos. Para que sí lo hagan, los instrumentos de medida deben estar construidos de otra manera. A partir de una Matriz del Bien Común, confeccionada sobre la base del cruzamiento de los valores mencionados con los grupos de contacto: proveedores, financiadores, empleados -incluyendo propietarios-, clientes, entorno social -tanto el puramente espacial como el temporal o intergeneracional-, a lo que se añaden algunos criterios negativos, por incumplimiento grave de normas y derechos internacionalmente reconocidos, cada empresa obtiene una nota final al realizar su Balance del Bien Común. En total 17 indicadores, que se desglosan en 68 sub-indicadores. Este valor, que resume hasta qué punto es genéricamente deseable la actuación de una empresa, queda a la vista de usuarios y consumidores, que ahora sí tienen todos los elementos de juicio para poder elegir entre la amplia oferta del mercado, aquellos que mejor se ajusten a sus criterios valorativos, para lo cual un semáforo de cinco colores que aparece en las etiquetas de los productos, nos avisa de hasta qué punto la empresa está contribuyendo al bienestar general. Quienes mejor nota obtienen, de más ventajas objetivas disfrutan. Entre ellas: menos impuestos, aranceles más bajos, créditos bancarios más baratos, prioridad en la compra o contratación pública y acuerdos con proyectos de investigación en universidades. Con este giro en la política fiscal, los productos fabricados y gestionados de manera ética, justa y ecológica serán más baratos, porque los no éticos, injustos, contaminantes o efímeros soportarán una mayor carga impositiva. La matriz se consensúa y actualiza democráticamente cada año. Por parte del Estado, el Producto del Bien Común se aproximaría bastante a medir la felicidad que proporciona la economía nacional al conjunto del país, por medio de una batería de al rededor de veinte indicadores. Todo esto supone un gran cambio en la legalidad, por la sustitución de: 


\section{LOS “BIENES DEMOCRÁTICOS» EN LA ECONOMÍA DEL BIEN COMÚN: CUATRO VÍAS PARA SU DELIMITACIÓN CONCEPTUAL}

a) la competencia (feroz) como modo regular de relación entre empresas o entidades por la confianza y la cooperación; y

b) la búsqueda y maximización del beneficio monetario por la búsqueda y maximización de la aportación al bien común.

Para ello, se habilitan disposiciones fiscales que premian a quienes mejor puntuación obtienen en su Balance del Bien Común, existiendo también penalizaciones para las organizaciones que causen daños o se dirijan en sentido contrario. La puntuación final del balance, obtenida aplicando la Matriz del Bien Común, no es sólo una cifra estática, por el contrario, ofrece una senda de perfeccionamiento continuo, en el que las ventajas eco-sociales promueven ventajas fiscales, que a su vez promueven de nuevo más ventajas eco-sociales, en una espiral que multiplica incentivos y motivación, con organizaciones privadas y públicas orientándose cada vez más hacia cuanto produce bienestar para todas las personas.

Con todo ello, la EBC redefine la manera en que culturalmente entendemos el éxito (económico) en nuestra sociedad. ${ }^{2} \mathrm{Y}$ para darle una nueva significación, declara un cuarteto de valores fundamentales que actúan como principios rectores a la hora de establecer valor económico: dignidad humana (1), solidaridad y justicia social (2), sostenibilidad ecológica (3) y co-decisión y transparencia (4). No deja de resultar paradójico que la presencia de estos indicadores y su carácter vinculante sean novedad en la economía del Estado, cuando no hemos hecho otra cosa que alinear las reglas del juego económico a los valores constitucionales y del derecho internacional que siempre estuvieron ahí. Otra grave contradicción del sistema capitalista se resuelve cuando el sistema de cambio el dinero- vuelve a su papel original como medio, dejando la posición de fin a lo que verdaderamente importa, el bien común.

El progreso de la EBC ha traspasado la frontera de las entidades empresariales para ayudar a las administraciones a orientarse de idéntico modo al bienestar general y acompañar a todo el proceso. En España, en 2013, la Asociación Federal Española para el Fomento de la Economía del Bien Común (AFEF-EBC) se propuso comenzar a trabajar en la adaptación de la matriz de manera que fuese viable medir la aportación al Bien Común por parte de los ayuntamientos. A principios de 2016 son más de una docena los que se han declarado institucionalmente a favor del proceso general de la EBC y algunos de ellos ya han incluido entre los criterios para la elección de empresas que se presentan a contratación o compra pública, presentar el Balance del Bien Común.

La EBC es un modelo abierto. Todos los principios que lo constituyen deben ser sometidos a un intenso debate democrático antes de ser convertidos en ley. No se auto-propone como el final de la Historia, sino sólo el paso que podemos dar ahora. Un paso lo suficientemente nuevo, como para

2.- Coincidiendo con esta visión, Jorge Riechmann señala que "el desarrollo sostenible no se ha traducido en una ética como un cuerpo de normas de conducta que reoriente los procesos económicos y políticos hacia una nueva racionalidad social y hacia formas sustentables de producción y de vida" (Riechmann y Abelda 2004:17). 
dar vida a las ansias de cambio y transformación que nuestra cultura necesita, y a la vez tan cercano, como para que nada de cuanto expone pueda considerarse una transgresión del modelo de mercado que, por ejemplo, la Unión Europea exige. Prueba de ello, en septiembre de 2015 una institución de la UE, el Comité Económico y Social Europeo (CESE) aprobó por abrumadora mayoría 3 un dictamen sobre la EBC que exige a la Comisión Europea avanzar en una hoja de ruta para la implementación concreta de los indicadores, etiquetado, un mercado interior y exterior y políticas encaminadas a ofrecer ventajas objetivas que recompensen a las empresas éticas, a partir de su Balance del Bien Común, entre otros elementos destacados, lo que da cuenta de las posibilidades reales con las que este modelo cuenta a la hora de ocupar una posición relevante, incluso dominante, en una zona del mundo sobre la que reposa nada menos que el $20 \%$ del peso de la economía mundial.

Intencionadamente, hemos dejado descolgados atrás dos de los elementos de fuerza de la EBC, Ios Bienes Comunales Democráticos, como los denomina Felber $(2010: 135-137,185,252,263)$ y las Comunidades del Bien Común (2010:225-227). De manera complementaria, las cuestiones de los ingresos solidarios (2010:89-90) o Renta Básica Universal (2010:249) junto con el valor de la naturaleza $(2010: 138,264)$ resultan también transformados en una propuesta integradora que los trasciende. La razón estriba en que vamos a presentar una propuesta de modificación que aglutina a todos estos elementos, dándoles un nuevo orden que creemos hace avanzar a la EBC aún más en sus propios objetivos. En las líneas siguientes, nos disponemos a clarificar, renovar y conceptuar términos como bienes comunales -commons-, bienes naturales, bienes neutros, bienes democráticos y bienes públicos, con los que hemos construido una nueva propuesta, íntimamente relacionada con -o mejor, que incorpora a- las Comunidades del Bien Común y con claras repercusiones de cara a avanzar en el diseño de la Renta Básica Universal o los ingresos solidarios, como los llama Felber. Para llegar hasta ellos, necesitamos antes retrotraernos hasta sus primeros cimientos, para discutir la cuestión de la dignidad, cuyo contenido está en la base de todos esos elementos.

3.- En su $510^{\circ}$ pleno de los días 16 y 17 de septiembre de 2015 (sesión del 17 de septiembre) el CESE aprobó por 144 votos a favor, 13 en contra y 11 abstenciones, el dictamen: La Economía del Bien Común: un modelo económico sostenible orientado a la cohesión social. En el apartado Conclusiones y recomendaciones, el CESE considera que la EBC es: "un modelo (...) concebido para incluirse en el marco jurídico europeo y nacional con el fin de avanzar hacia un mercado único europeo a través de una economía más ética basada en los valores europeos (...) refuerza los valores europeos y la cohesión social (...) fomenta un sistema económico responsable (...). En consonancia con la Estrategia Europa 2020, propone la transición hacia un "mercado europeo ético" que (...) será beneficioso para el medio ambiente. (...) podrá construirse mediante la aplicación de varias estrategias: Indicadores (...) como el Producto del Bien Común y los Balances del Bien Común (...) elaboración de políticas destinadas a reconocer a las empresas que contribuyen en mayor medida al bien común (...) las empresas europeas liderarán el mercado mundial ético y contribuirán a la promoción de los derechos humanos (...) El CESE exige a la Comisión Europea que, en el marco de la estrategia renovada de RSE, lleve a cabo un salto cualitativo que recompense (...) a aquellas empresas que puedan demostrar un mayor rendimiento ético. La crisis (...) ha puesto de manifiesto la escasa resiliencia del sistema económico y social de la UE. (...) La EBC es una herramienta para construir un sólido sistema social y económico que pueda dar a la sociedad civil europea "protección y desarrollo", que ayude a encontrar soluciones (...) La EBC es la manera de poner la resiliencia en el centro de los procesos (...)". Publicación oficial del CESE (2015). 


\section{2.- Objetivo: hacer de la comunidad un sujeto activo}

Que el primer artículo de los DDHH hable de fraternidad, justo después que lo hace de dignidad, libertad e igualdad, constituye un hecho para nosotros del todo remarcable. Nuestro ordenamiento social responde en cierto sentido a esa libertad con la institucionalización del llamado primer sector, el que rige las relaciones económicas (libre mercado). Podemos decir que a continuación responde a esa igualdad con una nueva institucionalización, la del llamado segundo sector, el que rige las relaciones gubernamentales, con el Estado velando por esa igualdad mediante su poder legislativo. Por último, la fraternidad ha encontrado su modo de institucionalización en las llamadas organizaciones del tercer sector (ONGs y asociaciones). En estas organizaciones la fraternidad ha sido entendida de una manera no estructural, recogiendo la fuerza de los nuevos movimientos sociales de finales de los sesenta -feminismo, ecologismo y pacifismo como principales ejes. Se han constituido como entidades benefactoras, con carácter caritativo, que palían las consecuencias más urgentes de las estructuras que causan daños eco-sociales, pero sin capacidad de hacer mella en esas estructuras, es decir, sin transformar sus causas. La enorme distancia que separa a los dos primeros sectores del tercero en cuanto a grado de institucionalización, puede detectarse dedicando una escueta mirada comparativa al listado de instituciones y aún más a la abundancia de niveles de organización. El Estado cuenta con administraciones que se organizan en varios niveles, que amplían su radio progresivamente: juntas de distrito, ayuntamientos, mancomunidades, diputaciones, comunidades autónomas, los ministerios de la administración central y, desde hace algunas décadas, también cuenta con el nivel comunitario (europeo), al que deberíamos añadir Naciones Unidas para tener una visión completa. Esto hace un total de ocho niveles. El mercado cuenta con empresas, algunas de ellas transnacionales, confederaciones de empresarios, organizaciones sindicales de trabajadores, la OCDE, y en el más alto nivel, el Foro Davos (y otros), el G7, la Organización Mundial del Comercio, el Banco Mundial y el Fondo Monetario Internacional. Al menos cuatro niveles y un poder tan grande que a menudo la UE debe establecer medidas anti-monopolio. Para poder comparar, nos preguntamos cuáles serían las instituciones equivalentes en el tercer sector. La sociedad civil cuenta ciertamente con una institución tan poderosa como histórica: la familia. La familia se comporta como una estructura de base, efectivamente basada en las relaciones de fraternidad y como tal, está preparada para soportar las mayores tensiones provocadas por los otros dos sectores. En los momentos más críticos la sociedad mantiene unos mínimos de resiliencia grupal gracias a ella. Vivimos por varios años en España la constatación de cómo con un sólo salario o incluso ninguno, familias enteras pasan la travesía del desierto de los periodos de recesión económica en condiciones que podemos llamar de subsistencia. Pero ¿qué otras estructuras que den continuidad institucional a la sociedad civil se elevan más allá de la familia en tanto que estructura comunitaria? Las comunidades 0 asociaciones de vecinos constituirían el siguiente nivel natural. Desde el punto de vista legal, el volumen de los contenidos es cuantitativamente profuso 4 ,

4.- El texto de la Ley de la propiedad horizontal nos puede servir para una primera medida de dicho volumen. 
pero desde el punto de vista del funcionamiento de las comunidades de ámbito vecinal, abundan las carencias en las relaciones sociales -el medio de su actividad natural-, mermadas cuando no aniquiladas, como es sabido, en gran parte de las ocasiones. De modo que, sin contar la familia -clamorosamente insuficiente para alcanzar una masa crítica que pueda organizar una gestión propia de la sociedad civil- la respuesta va a ser cero, tanto si contabilizamos las instituciones como sus niveles. Únicamente funciona el primer nivel y ahí se acaba todo. En la familia comienza y termina el diseño de la arquitectura institucional del tercer sector en nuestra sociedad convencional. El sector viene definido precisamente por las relaciones sociales y por la fraternidad como valor más propio. Este análisis nos lleva directa e inexorablemente a la necesidad de comprender bien el funcionamiento de las comunidades y, dentro de un esquema para la economía que por primera vez trabaja de abajo arriba como es la EBC, nos ayuda a entender la enorme importancia de abordar, al tiempo que unas empresas y unos ayuntamientos, unas Comunidades del Bien Común.

\section{Comunidades del Bien Común, comunidades resilientes}

La mayoría de las veces que hemos abordado el asunto de las comunidades en público, con ocasión de encuentros, congresos o jornadas donde hemos explicado los detalles de la puesta en marcha en España de la EBC, se ha suscitado una pregunta: ¿de qué clase de comunidades hablamos? Es aparentemente fácil de entender que entre los asistentes no haga acto de presencia una idea mental inequívoca acerca de a qué comunidad nos podemos estar refiriendo. A falta de un examen más sistemático que recoja lo que brota en las mentes, podemos aportar algunas de las imágenes que ha sugerido la palabra. A algunas personas, comunidad parece traerles a la memoria quienes se reúnen por relación de afecto, como los grupos de amigos que programan actividades de tiempo libre. A otros, les evoca quienes proyectan colectivamente un tipo especial de compra o acceso a un tipo de producto para el que el mercado no dispone de oferta, los florecientes grupos de consumo, como por ejemplo, los que acuerdan un sistema para consumir alimentos de agricultores cercanos, o para el acceso a internet en zonas rurales donde a las empresas suministradoras no les resulta rentable invertir. Hay quien piensa en colectivos que se reúnen para compartir vehículo o alojamiento en los viajes o para cualquier otro tipo de consumo colaborativo. Con frecuencia vienen a la memoria las agrupaciones de personas que deciden dejar la ciudad para comenzar una nueva vida en el campo, formando asentamientos de los llamados neo-rurales. Reconociendo en todos ellos elementos comunitarios importantes que más adelante vamos a recuperar, es cierto que sólo en el último caso las personas experimentan la comunidad de una manera completa o sustancial en sus vidas. De todas las representaciones aducidas es la que más se acerca. Sin embargo, nuestra primera advertencia ha venido siendo explicar que no las restringimos a comunidades nuevas que se establecen con la intención de poder romper con el sistema. Ese caso nos adentraría en la estrategia de escape que ya habíamos dejado a un lado al comienzo de esta exposición. 


\section{LOS “BIENES DEMOCRÁTICOS» EN LA ECONOMÍA DEL BIEN COMÚN: CUATRO VÍAS PARA SU DELIMITACIÓN CONCEPTUAL}

Es sabido que en los próximos años, un cincuenta por ciento de la población de todo el globo residirá en las ciudades, llegando al sesenta por ciento la previsión para 2030.5 Es en la ciudad donde los seres humanos experimentan la condición de ciudadanía, en plenitud de la función política ${ }^{6}$, con todos los derechos civiles y políticos. Hemos de pensar pues, en desarrollos que no condenen a la población al exilio, elección que Sócrates se negó a considerar, ni tan siquiera como alternativa a la cicuta. Por lo tanto, la apuesta no va a residir en apartarse de la sociedad; "se trata más bien de emprender el cambio social desde el corazón mismo de las comunidades ya establecidas" (Azkarraga 2014:97).

Este mismo hecho, la falta de claridad en la idea de comunidad suscitada, no hace sino confirmar y poner una vez más de manifiesto cómo la sociedad civil permanece al margen de las instituciones de fuerza. Una cuestión para nosotros fundamental es cómo las sociedades humanas desarrolladas han construido todo un sistema alrededor de un eje de tensión social, política y también económica que se balancea entre dos extremos, a saber, el mercado y el Estado. A consecuencia de esto, el marco de posibilidades de cuanto puede concebirse está siempre a uno u otro lado, ejerciendo el efecto de imantar cuanto se acerca, con la zona central, intermedia, que queda para los casos menos polarizados. La importancia de aumentar el número de partes hasta tres, por la inclusión de la sociedad civil, no obedece únicamente a un reconocimiento de esta tercera pata de nuestro ordenamiento. Además de esto, el mero hecho de aumentar formalmente las partes a tres, multiplica considerablemente las combinaciones posibles a la hora de diseñar formatos o figuras legales de empresa (sociedades mercantiles y otras) y lo que es más importante, abre un inmenso espacio interior: un plano. Más allá de la recta surge el plano como la siguiente figura geométrica, definida por tres puntos. En este nuevo espacio que inauguramos al dar entrada a la sociedad civil como tercer polo sistémico, toda propuesta podrá ser situada, con amplio margen de ubicación y maniobra. Nuestra sociedad se constituye como un complejo donde la esfera del Estado tiene un lugar propio, con sus entidades gubernamentales, como ayuntamientos, diputaciones, comunidades autónomas y demás administraciones públicas, de las que todos poseemos experiencia y conciencia. Lo mismo puede decirse del mercado. Las relaciones comerciales se ponen de manifiesto a diario en la vida de cualquier persona, en cualquier lugar del mundo. En cambio, la sociedad civil apenas se pone de manifiesto por sí misma, más allá de la negación de su pertenencia a las otras dos esferas. Se denominan así sus entidades organizaciones no lucrativas, como negación de su pertenencia al primer sector, a la vez que no gubernamentales, como negación de su pertenencia al segundo. El tejido asociativo es mayoritariamente mencionado y reconocido como ONG durante y después del boom de mediados de los noventa, con especial mención a las asociaciones de desarrollo (ONGD). Esto subraya la escasa institucionalización que posee en nuestro orden social la sociedad civil, con un papel muy secundario o enfocado hacia el exterior, especialmente si se la compara con las otras dos esferas. Es a partir de esta ausencia de función y sentido, que nuestra concepción de comunidad se eleva para constituirse en una pieza fundamental del engranaje necesario para poner en marcha una economía del bien común, a partir de la revitalización de sus posibilidades institucionales.

5.- Según datos publicados en la web del Banco Mundial: <www.bancomundial.org/temas/cities/datos.htm>. Libro l.)

6.- Recuperamos aquí, con Aristóteles, la noción de ciudadano, partícipe de la política, de la vida de la polis -zoon politikón (La Política, 
Ya tenemos pues, resuelto el significado de la comunidad. Queda clarificado que las comunidades son las entidades donde las relaciones sociales que dan desarrollo al valor de la fraternidad se estructuran, de manera regular, dentro de la amplia esfera de la Sociedad Civil. Podemos añadir que esas comunidades se disponen en una escala de niveles que parte del hogar, como suma de la estructura familiar y el espacio geográfico en el que se sitúa dentro del espacio público: la vivienda. El siguiente nivel lo constituyen las agrupaciones de hogares en comunidades de vecinos, geográficamente localizables en urbanizaciones. El siguiente escalón lo conformarían los barrios, como unidades compuestas por la reunión de las entidades del anterior nivel. Las localidades agruparían los barrios, como las comarcas aparecen por la agrupación de aquellas. Las provincias, igualmente reúnen a las comarcas, mientras las regiones suman provincias en el penúltimo peldaño, que concluye en la nación. La nación, de la raíz natio ${ }^{7}$ revive la idea de que nacional es quien ha nacido -de la raíz nasci- en el seno de una gran familia. Una comunidad no biológica, sino cultural, que da el paso de convertirse en una comunidad política, al reconocerse a sí misma como sujeto político, estableciéndose entonces la comunidad nacional. De este modo, la nación comporta la máxima amplitud de los límites culturales de la familia. Familia, cuyos lazos biológicos han sido llevados a su máxima extensión, por actuación de lazos políticos que se reconocen sobre una base mítica, como una especie de parentesco remoto, situado en el origen.

Pero todo ello lo absorbe el Estado, vaciando de instituciones a la comunidad en tanto que tal. Cada uno de los niveles posibles $\{. .$. comarca, provincia, región... $\}$ son representados por sus respectivas administraciones \{...mancomunidad, diputación, comunidad autónoma.... . El hogar es a todas luces una estructura insuficiente, recordemos, como masa crítica que pueda ocuparse de un papel fundamental, equiparable a como las empresas 0 las administraciones se ocupan de las relaciones comerciales y gubernamentales. Si la EBC transcurre como modelo bottom-up, de abajo arriba, el gran reto consiste en pertrechar un proceso de recomposición social que devuelva a las comunidades (vecinales y siguientes) la capacidad de articular relaciones socialmente productivas de su propio ámbito, que estando regido por el valor de la fraternidad, podemos describir como, de relaciones basadas en la confianza, la cooperación, la ayuda y el reconocimiento mutuos. Esto puede resumirse en que la comunidad es algo más que un simple colectivo: un colectivo que adquiere comunitas o sentido de comunidad porque hay lazos (extrafamiliares) de convivencia por entre su entramado. Un aumento de la calidad humana, entendida como calidad y cantidad de bienes relacionales, será, entre tanto, obligado en muchas de ellas, antes de transformarse en una Comunidad del Bien Común 8 . La razón se encuentra en que, más allá de la importancia que las relaciones comunitarias poseen por sí mismas, todas las operaciones que harán funcionar la comunidad como comunidad del bien común partirán en toda circunstancia de las posibilidades ofrecidas por la calidad de las interacciones entre sus componentes. La talla de esas relaciones marcará el primer y más acuciante límite a su potencial. Recogiendo las palabras de Juan Torres, necesitamos un proyecto civilizatorio que reconstruya espacios de encuentro para potenciar dichos acercamientos en un espacio que los arrope y pensar formas

7.- Según el Diccionario Etimológico de Chile <http://etimologias.dechile.net/?nacio.n>.

8.- El concepto de Comunidad del Bien Común aparece por primera vez en el manual de Felber (2010:23) y es someramente explorado en un apartado específico (225-7). 


\section{LOS “BIENES DEMOCRÁTICOS» EN LA ECONOMÍA DEL BIEN COMÚN: CUATRO VÍAS PARA SU DELIMITACIÓN CONCEPTUAL}

alternativas de producir que nos permitan producir y consumir de otra manera. En pocas palabras necesitamos crear comunidad para gestionar lo común en común. Y la primera de las producciones ha de ser la producción de relaciones para el buen vivir ${ }^{9}$.

Para entrenar las disposiciones corporales que nos llevan a producir relaciones en el marco de unas estructuras objetivas representadas por las comunidades de vecinos, contamos con propuestas de nueva generación como la Biodanza, en la que se trabaja el campo emocional implicado en las interacciones humanas tanto individualmente como entre pares o en grupo, o la Facilitación de Grupos, para encaminar el proceso comunicativo y participativo grupal de cara a la toma de decisiones y la organización de la acción colectiva. Desde nuestro punto de vista, reconocemos el gran potencial instrumental que estas disciplinas ofrecen como herramientas para la constitución de grupos emocionalmente sanos y con buena salud en su estructura y funcionamiento. Estos instrumentos aportan un gran valor a la hora de sensibilizar y concienciar a las personas, convirtiéndolas en actores o agentes de paz ${ }^{10}$, quienes habrán de poner en marcha estructuras de paz, como veremos enseguida. Es conveniente señalar que, una vez transformada la comunidad de vecinos y replicado el proceso en varias de ellas geográficamente agrupadas, habrá ocasión de elevar el proceso al siguiente nivel, es decir, aparecerá la posibilidad de construir Barrios del Bien Común, como el paso natural evolutivo, que una vez logrado se podrá encaminar a su vez a la consecución de Localidades del Bien Común, que nuevamente tendrán ocasión de componer Comarcas del Bien Común, toda vez que el proceso puede repetirse recursivamente, siguiendo un modelo que en cierto modo recuerda a los fractales.

Ahora que ya tenemos claro lo que entendemos por comunidad, la siguiente pregunta sería: ¿cómo convertir nuestras comunidades (de vecinos y siguientes) en comunidades del bien común? ¿Qué diseño podría reorientarlas? Para responder a esto, primeramente hay que tener claro qué fin es el que se persigue con esa orientación; si es suficiente con una gestión siguiendo el modelo aplicado para empresas, como por ejemplo "compras con sentido ético". En este sentido recogemos lo que Felber ha descrito como actividades propias de una Comunidad del Bien Común (Felber 2010:225-7):

1. apoyar la iniciativa de la EBC e inscribirse en la web como comunidad partidaria

2. Implementar su Balance del Bien Común (BBC) comunitario

3. Invitar a las empresas residentes a elaborar el BBC empresarial

4. Desarrollar el Índice Comunitario del Bien Común y redactar un Índice de Calidad de Vida

5. Acoger la celebración de convenciones del Bien Común

6. Implicarse en la creación del siguiente nivel (Barrio del Bien Común, como proponemos aquí)

La propuesta que justifica esta investigación consiste, de manera nuclear, en añadir un elemento de gran importancia a los seis puntos arriba expuestos por Felber que añade peso específico institu-

9.- Conferencia pronunciada por Juan Torres en el Ateneo de Málaga en agosto de 2015, con ocasión de la creación de la candidatura de confluencia ciudadana Ahora en Común.

10.- Galtung engloba dentro de su concepto de Paz Directa las acciones de paz encaminadas a sensibilizar, concienciar, y en general formar a las personas como agentes transformadores para el conflicto (conflict workers). 
cional a la sociedad civil. Pero para guiar nuestra búsqueda hacia ese elemento necesitamos de un principio que nos guíe, que opere como causa final, es decir, que determine la elección del comienzo para proporcionar un resultado esperado. Esto quiere decir que antes de proseguir, necesitamos tener en mente el principio que nos ha de llevar al esclarecimiento de ese papel fundamental que una comunidad debe ostentar para considerarse como orientada al Bien Común, bajo el prisma de la pretensión de un sistema que institucionalice la Sociedad Civil con la misma fuerza que lo está actualmente el Estado y el mercado, como venimos sosteniendo hasta el momento. Y para encontrar ese principio rector del ámbito comunitario, nos vamos a ayudar de una analogía con la empresa (o la administración) como elemento comparativo, de tal manera que podamos establecer entre ambos un paralelismo o una ecuación, de la cual podamos despejar la incógnita del esperado principio.

Una empresa es la entidad de base dentro de la esfera del mercado, como la comunidad lo es en el interior de la Sociedad Civil. El mercado constituye el escenario natural para las relaciones económicas, como la Sociedad Civil aporta el escenario natural para las relaciones sociales. A partir de estas señas, nos encontramos en buenas condiciones para comenzar a plantearnos la pregunta clave: ¿Cuándo sobrevive una empresa? La respuesta es irrebatible: una empresa sobrevive cuando es rentable/viable, pues de lo contrario quiebra. Y con esta aseveración nos planteamos cuál es su paralelo para la comunidad. Ésa es la pregunta con la que obtendremos el criterio que nos llevará al diseño de comunidad que pretendemos.

Para poder encontrar la palabra que nos defina con exactitud qué condición debe cumplir por encima de todo una comunidad para sobrevivir como tal, podemos comenzar por apuntar algunos rasgos que nuestro principio buscado debiera incluir.

1. Si la Sociedad Civil es una esfera enfrentable a las otras dos (mercado y Estado), debería tener autonomía suficiente para definir sus propias reglas y encontrar un campo de actuación propio en el que las comunidades pudieran asegurar su supervivencia.

2. Esta supervivencia debería realizarse en términos independientes o autónomos de estructuras exteriores, o dicho de otro modo, debería encontrar la manera de depender más de sí misma y menos de los otros sectores (empresas y administraciones).

Una sociedad civil que mira al Estado y al mercado peer to peer, es decir, desde una posición entre iguales, determina para sí misma una autonomía proporcional a la hora de definir su existencia, o lo que es lo mismo, su supervivencia. Por lo tanto, sus condiciones materiales de existencia han de caracterizarse por atributos como autoafirmación, autonomía, autosuficiencia e iniciativa. A partir de estos rasgos, creemos suficientemente acreditado que el principio fundamental y fundacional que ha de dirigir la manera de entender una Comunidad del Bien Común es el de resiliencia11.

11.- Aunque reiteradamente usamos el término resiliencia, nos referimos a resiliencia social o grupal, incluso más concretamente a resiliencia comunitaria, que dejamos en una sola palabra por pura economía lingüística. Mientras que el primero ha surgido de la Psicología, el segundo supone una derivación de aquel para volverlo operativo en el terreno de la sociología, la antropología y otras ciencias sociales. 


\section{LOS “BIENES DEMOCRÁTICOS» EN LA ECONOMÍA DEL BIEN COMÚN: CUATRO VÍAS PARA SU DELIMITACIÓN CONCEPTUAL}

En el terreno de la psicología se ha venido entendiendo la resiliencia como una capacidad en personas, grupos y comunidades -capacidad incluso de alcance universal (Gil 2014:194)- para enfrentarse y sobreponerse a las adversidades, con flexibilidad y siendo transformadas por ellas, encontrando nuevos caminos, nuevas posibilidades (Forés, Grané, Ollé 2012), construyendo nuevas interpretaciones que resignifican vidas y siempre a partir de las potencialidades y los recursos propios. Subrayamos que frente a otras salidas alternativas a ella como desistir o resistir, la resiliencia incluye el importante matiz de la transformación creadora. La energía de la adversidad se aprovecha para a partir de ella crear una nueva respuesta más adaptativa ${ }^{12}$, cargada de futuro. No puede resultar entonces extraño que la creatividad, la iniciativa, la autonomía, la empatía sean frecuentemente descritos como sus pilares (Suárez 2001). Los procesos de resiliencia tienen que ver en un principio con resistir, desde luego, pero van mucho más allá. La resistencia es terca, repetitiva, poco flexible. La persona resiliente, en cambio, es capaz de visualizar otros mundos posibles y dirigir la mirada hacia ellos. Observa las potencialidades y trabaja las fortalezas. De algo doloroso puede sacar algo maravilloso, paradójicamente. Es capaz de releer su propia historia para dejar de verse como víctima y comenzar a sentirse afortunado. La respuesta resiliente no se ancla pues al dolor, sino que resurge a partir de él. Todo ese cambio de rumbo exige un cambio de mirada: pararnos a observar cómo podemos girarnos hacia el lado de las potencialidades.

El párrafo anterior nos acerca al universo de la resiliencia aplicable a los comportamientos individuales, desde un punto de vista psicológico. Pero necesitamos trasladar el concepto de resiliencia al ámbito de la grupalidad, para poder abordar cómo y cuáles en concreto habrían de ser las respuestas resilientes de nuestra comunidad. En la misma linea que señala Madariaga, hemos de "entender cómo generar un marco que haga posible la construcción social de la resiliencia como respuesta a los retos a los que nos enfrentamos actualmente como sociedad" (2014:14). Para ello, y concediendo al factor ecológico o ecosistémico una presencia e importancia centrales dentro de cualquier construcción social de la resiliencia que permita a la sociedad civil sobreponerse de la crisis sistémica actual, nos apoyamos en la aproximación sobre resiliencia que realiza Azkarraga, que entiende la resiliencia comunitaria como el mecanismo para abordar una "transformación sustancial del actual metabolismo sociocultural, que tienda a simplificarlo, para que la satisfacción de las necesidades humanas básicas requiera de menos recursos naturales" (Azkarraga 2014:91-92) y aporte soluciones a la crisis ecológica:

La relocalización de las actividades humanas es inevitable (...). Las claves de futuro consistirán en prepararse y caminar hacia territorios más autosuficientes, con estructuras descentralizadas y de menor escala, y con mayores niveles de auto-organización comunitaria. Una comunidad con un alto grado de autosuficiencia en la satisfacción de sus necesidades estará mejor preparada que aquellas sociedades que dependan de sistemas globalizados para satisfacer las necesidades básicas de energía, transporte, vivienda, sanidad o alimentación. Hablamos, por tanto, de rupturas importantes con respecto al actual modelo de desarrollo y de sociedad (...)

12.- Sirva la metáfora del yudoka que al recibir un golpe utiliza la energía del gesto de su contrincante para caer o rodar de la manera más conveniente, o efectuar un movimiento nuevo como respuesta. Es imagen prototípica de resiliencia la planta que consigue crecer brotando en una pared de piedra. 
El mismo autor define relocalización como el proceso por el cual un determinado agregado humano se libera de su dependencia con respecto a la economía global, de manera que produce una parte creciente de sus alimentos, energía, bienes y servicios que consume (Azkarraga 2014:92). Nosotros especificamos que esta independencia no es sólo deseable respecto de las grandes empresas (en la práctica, oligopolios) sino también de las posibles administraciones, de tal manera que la comunidad pase a depender lo menos posible de cualquier entidad exterior y lo más posible de sí misma, si bien no para cualquier sector, sino en lo tocante a los bienes más indispensables, sobreentendiendo que cuando hablamos aquí de comunidad lo hacemos en un sentido amplio, dentro de una red, junto a otras comunidades, en una arquitectura organizativa que pudiera tener su techo en el conjunto de la región, como estructura propia para el tejido de la sociedad civil. Coincidimos plenamente con Azkarraga, además, en que poner la esperanza en los extremos superior (cambio de Gobierno) o inferior (cambio interior de cada persona) constituyen salidas en falso en el sentido de que si bien son pasos igualmente necesarios, no dotan de soberanía para la acción en el primer caso, o de masa crítica suficiente para el segundo, a la hora de definir realmente el proceso transformativo de abajo arriba (Azkarraga 2014:97).

Antes de continuar hemos de especificar que el tipo de resiliencia que nos transportará al conjunto de transformaciones que necesitamos para una comunidad posee otros rasgos importantes. Por ejemplo, contiene un rasgo de anticipación que no responde a una mera estrategia defensiva ante la emergencia -algo que no se contemplaba en los primeros estudios sobre resiliencia- sino de construcción estructural de mayores niveles de bienestar (Reich, Zautra y Hall 2010). Anticipación que contiene a su vez un matiz fresco, optimista y gratificante que exploramos con ayuda de Forés, Grané y Ollé como capacidad de convertir las amenazas en oportunidades antes de que hagan acto de presencia, lo que llaman "ensayar la resiliencia" (2014:232). También consideramos esencial una cualidad fundamental que ellas llaman resiliencia generativa o proactiva, es decir, que se adelanta proponiendo transformaciones "antes de que el cambio sea una necesidad". Tal capacidad genera posibilidades mediante tres vectores: apreciar y potenciar lo que funciona, construir y recrear espacios para las oportunidades y transformar y fortalecer los vínculos relacionales (2014:234).

En la actitud resiliente, el sentido aparece con el cambio de mirada. Recordemos que toda respuesta resiliente comienza con un giro que nos coloca a nosotros, la comunidad, en el centro, dándonos la gran oportunidad de ejercer la responsabilidad de ser la variable independiente que arranque el motor del cambio. Si las comunidades tomáramos directamente a nuestro cargo la gestión de cuanto resulta verdaderamente vital para la propia subsistencia, ¿Escogeríamos alimentos tóxicos que al mismo tiempo degradasen la economía de los agricultores? ¿Seríamos víctimas de desahucios cuando no alcanzáramos a pagar hipotecas abusivas? ¿Tendríamos que pagar cuotas desorbitadas por acceder a internet? ¿Nos conformaríamos con facturas de suministro eléctrico tan opacas como disparatadas en precio, mientras nos impiden aprovechar fuentes de energía ubicua e inagotable mediante autoconsumo? 


\section{LOS “BIENES DEMOCRÁTICOS» EN LA ECONOMÍA DEL BIEN COMÚN: CUATRO VÍAS PARA SU DELIMITACIÓN CONCEPTUAL}

Pero volvamos al punto de partida para la acción desde esta actitud generativa. Su anclaje no puede hacerse más que a partir de comunidades que tomen conciencia de su propio papel: ¿Y si en lugar de posar la mirada sobre las empresas del Oligopolio que nos maltrata con condiciones deleznables; 0 en la actuación -complementaria- de los sucesivos gobiernos que mantienen con ellas relaciones que propician escenarios de corrupción estructural...? ¿Y si en lugar de poner la atención en todo ello nos girásemos hacia la enorme potencialidad que posee nuestra comunidad para iniciar una andadura de consciencia plena utilizando todas las fortalezas que para ello posee? ¿Y si comenzamos por tener más y mejores relaciones dentro de la comunidad, creando confianza, sentido de compartir, de colaboración y de cooperación para poder desarrollar tales respuestas de resiliencia? ¿De qué manera tendrían las comunidades un papel trascendente que cumplir dentro de un sistema tripartito, cuando la Sociedad Civil se encuentre ya tan institucionalizada como el mercado o el Estado, con unas relaciones sociales promocionando la fraternidad de manera equiparable a como el mercado promociona la libertad mediante las relaciones comerciales o el Estado la equivalencia entre todas las personas a través de las acciones gubernamentales o legislativas? Para responder a estas peguntas nos embarcamos en la propuesta de transformación de las comunidades, navegando ya con la resiliencia, en particular la resiliencia generativa, como timón.

\section{3.- Conclusión: los bienes democráticos como concepto económico}

Los atributos -compatibles con la experiencia resiliente- antes mencionados de autoafirmación, autonomía, autosuficiencia e iniciativa nos descubren el camino hacia las respuestas. Una comunidad, para ser generativamente resiliente habrá de tomar la iniciativa y dotarse de manera autónoma de la mayor cota posible de suficiencia en la gestión de los bienes más indispensables, aquellos bienes de los que, como ya hemos explicado, la dignidad depende en su vertiente material; creándose un bucle cerrado que provee y recepciona a la vez condiciones de vida mínimamente aceptables. Una gestión de estos bienes de la que podamos decir que se configura como respuesta resiliente promoverá su producción y consumo sin dejarlos depender de instituciones externas, ya fueren privadas 0 públicas, tomando así la comunidad las riendas para dirigir, controlar y gestionar estos bienes de manera más directa.

Definimos los Bienes Democráticos como aquellos sectores económicos que, reunidos en una categoría jurídica propia que el Estado declara como tal ${ }^{13}$, proporcionan la componente material para la satisfacción de las necesidades humanas básicas, determinando las condiciones materiales míni-

13.- Aconsejamos desterrar la usual denominación de sectores estratégicos, que se corresponde con los intereses materiales y simbólicos de los agentes del mercado, reflejo de la importancia que éstos depositan en ellos, al tratarse de bienes irrenunciables para la ciudadanía, por su carácter básico y necesario para la vida humana. 
mas y aceptables de dignidad y derechos humanos, mediante una gestión de máximos democráticos, a través de los principios de cooperación sin interés de lucro, organización empresarial desde el lado de la demanda e inclusión de toda la cadena de valor, esencialmente producción, trabajo y consumo, con la mayor eficiencia y transparencia y el menor impacto sobre el medio ambiente, conformándose como un sistema de autoconsumo generalizado para el tramo ${ }^{14}$ más básico de estos bienes dentro de un marco de resiliencia comunitaria. A la espera de suficiente consenso, éstos podrían alternativamente denominarse Bienes Fundacionales, por su papel fundacional en las sociedades humanas, disparando las principales etapas de la civilización; Bienes Constituyentes, por su ubicación natural entre los derechos fundamentales de las constituciones; y finalmente, Bienes Comunitarios, por tener -más allá de lo privado y lo público- su sujeto de gestión en la comunidad. Incluimos algunos de sus rasgos más importantes:

a) Algunos de los Bienes Democráticos los compartimos con cualquier forma de vida, otros son específicamente humanos, marcando hitos a lo largo de la Historia (discontinuidades).

b) Los Bienes Democráticos vienen prefigurados en la naturaleza como funciones orgánicas esenciales (nutrición celular, aparato fonador y acústico, aparato locomotor, homeostasis...)

c) La Constitución debe declarar su existencia, consagrando como derecho fundamental el paquete mínimo de sectores: el derecho inquebrantable a los Bienes Democráticos.

d) La ley ha de desarrollar este derecho mediante la creación de una forma jurídica propia que establezca un modo de gestión para estos sectores, de acuerdo con su naturaleza y con especificaciones sector por sector (por ejemplo, S. Coop. de Bienes Democráticos).

e) Las comunidades han de prestar espacios de encuentro no-comerciales/nogubernamentales para la gestión y entrega de estos bienes, optimizándose así en conjunto su eficiencia.

En su denominación, la palabra democráticos se debe a varias razones, que a continuación detallamos:

a) Por su universalidad: Toda persona necesita en condiciones normales el conjunto completo de estos bienes para poder vivir, o para poder vivir dignamente.

b) Por su igual distribución: Toda persona necesita en condiciones normales idénticas proporciones (el tramo básico) de dichos bienes, con independencia del nivel socioeconómico u otra condición personal.

14.- La cuestión del tramo posee una importancia capital dentro del concepto de bienes democráticos, si bien no será abordada hasta el trabajo específicamente económico que desarrollaremos en una publicación posterior. Sirva por el momento la escueta reflexión de que cada uno de los sectores involucrados debe tener asignado un límite superior que define su uso en tanto que bien democrático. Por ejemplo, para el agua se baraja la posibilidad de 120 litros por persona y día. Para establecer al detalle estos límites estamos trabajando con un panel de expertos, que ayudará a determinarlos previo debate. 


\section{LOS “BIENES DEMOCRÁTICOS» EN LA ECONOMÍA DEL BIEN COMÚN: CUATRO VÍAS PARA SU DELIMITACIÓN CONCEPTUAL}

c) Por su modo de gestión: Todas las personas poseen el mismo poder. La propiedad y el poder en la empresa que los gestiona está repartida por igual entre todas las personas socias. Si ocurriera que la cultura evolucionase permitiendo modos organizativos más democráticos, ello obligaría a modificar la ley para incluirlos, de tal manera que siempre se cumpla que nada más democrático en nuestra sociedad, que el modo de gestión establecido para los Bienes Democráticos.

Para identificar los Bienes Democráticos, usaremos la guía que Galtung (1977:10) propone, un escueto listado de necesidades básicas, subyacentes dentro de toda conflictividad humana, que ha estudiado durante seis décadas: supervivencia, bienestar, libertad e identidad. ${ }^{15}$ Con ellas nos disponemos a cartografiar el mapa de los sectores económicos que definen la existencia específicamente humana y su dignidad correlativa. Estas cuatro necesidades básicas se relacionan con cuatro estratos de funciones que son cubiertas con determinados sectores económicos.

1. La necesidad de seguridad/supervivencia se relaciona con la función de infraestructura.

2. La necesidad de bienestar se relaciona con la función de suministros.

3. La necesidad de libertad se relaciona con la función de comunicación/comunicaciones.

4. La necesidad de identidad se relaciona con la función de reproducción y perpetuación.

Cada uno de los estratos funcionales despliega un doblete de sectores que lo materializa. La infraestructura se desdobla en vivienda y agro-ecología comunitaria. Es decir, un hogar que recupera la función alimentaria y de abrigo al proporcionar agro-alimentos y agro-textiles, mediante un sistema de permacultura, que puede apoyarse sobre una distribución en cascada que comienza en viveros periurbanos, que proveen de especies a viveros urbanos, que a su vez pueden proveer a las comunidades, que integran en ellas un paisaje comestible que incluye a las llamadas plantas multifuncionales, con funcionalidades más específicas, de todo tipo. ${ }^{16}$ De este modo, la ordenación del territorio urbano incluye soluciones de sector primario, al proveer tanto de infraestructura de abrigo (viviendas) como para el alimento (horto-frutícola) y el vestido (agro-textiles: algodón, lino, cáñamo...). Estos bienes nos proporcionan la base de seguridad que necesitamos en primerísimo lugar: satisfacer la función de infraestructura que se encuentra asociada a la necesidad básica de seguridad o supervivencia.

Identificamos los suministros vitales de esa infraestructura con agua y energía eléctrica, que dan satisfacción a las necesidades primordiales de los seres humanos (hidratación, aseo...), a la propia infraestructura agroecológica antes mencionada (riego) y a su tecnología, que poco a poco va sofisticándose en la forma dominante de dispositivos electrónicos, esto es, que necesitan electricidad como fuente de alimentación, dentro de un contexto cultural marcado por este tipo de tecnologías. Estos

15.- Por razones de espacio no hemos abordado aquí el análisis de los conceptos de necesidades y de necesidades básicas, con la profundidad necesaria e insistiendo en sus relaciones con los bienes democráticos. De todo ello tendremos oportunidad de dar cuenta en una publicación posterior, en la que desarrollamos la vía específicamente económica.

16.- Relacionadas con las plantas multifuncionales (PlaM) se pueden citar una gran cantidad y variedad de funciones. Entre ellas, destacamos: la elaboración de cosméticos y productos de limpieza, el tratamiento de la tierra de cultivo o de las plagas y en particular, las plantas medicinales o con un valor específico para la salud. 
suministros necesarios para la infraestructura proporcionan a los seres humanos el entorno de satisfacción mínimamente aceptable para cubrir la necesidad de bienestar.

Las necesidades de supervivencia y bienestar "nos anclan a tierra". Pero súbitamente, los humanos experimentamos una tercera necesidad básica, relacionada con una actitud expansiva: la libertad. Necesidad que se satisface con la doble función de las comunicaciones: la libertad de expresión y la libertad de movimiento. Las comunicaciones se desdoblan, en primer lugar, en las informativas, cuya satisfacción material básica sucede al disponer ininterrumpidamente de conexión a internet, dando una extensión completa a la funcionalidad natural del aparato fonador y acústico en un contexto de sociedad-red. Con ellas experimentamos la libertad más inmediata, la libertad de expresión, que incluye, por extensión: investigación, difusión, acceso a bienes culturales, ciencia, arte, literatura y en general a todo contenido informativo, incluyendo el reservorio de carácter personal que supone la nube, es decir, dando posibilidad real a muchos de los derechos fundamentales intangibles. Las otras comunicaciones son las que nos permiten desplazar el propio cuerpo más allá de las posibilidades naturales del aparato locomotor, es decir, los medios de transporte, con los que experimentamos la libertad de movimiento, que en su forma más elemental identificamos con el desplazamiento por el hábitat de cualquier civilización: la ciudad. Hablamos, por tanto, de movilidad urbana. Éstas son las primeras libertades relacionadas con bienes, las más íntimas y básicas, también precursoras 0 posibilitadoras de otras, expresadas junto con las condiciones materiales para su realización efectiva.

La reproducción o perpetuación de los sistemas humanos comprende en todo caso la doble dimensión humana: individual y colectiva. La identidad individual se mantiene mediante el acceso a los procesos de atención a la salud, es decir, mediante el sistema sanitario. La identidad colectiva se mantiene mediante el acceso a los procesos de transmisión-adquisición de la cultura, es decir, mediante el sistema educativo. La identidad como necesidad básica queda, por lo tanto, materialmente soportada en estos dos sistemas. Con ellos reproducimos porque volvemos a producir todo el sistema de bienes indispensables. Cerramos así el círculo y con estos bienes democráticos volvemos al comienzo de la argumentación.

\section{Cuatro vías para delimitar conceptualmente los Bienes Democráticos}

La dignidad es, como hemos visto, el punto de partida para el establecimiento de los Bienes Democráticos. La resiliencia representa el timón que nos guía para encontrarlos y definir su modo de gestión. Las necesidades humanas básicas nos definen las funciones que cumplen. Las comunidades aportan el sujeto de la acción. A través de los recientes avances en la economía (bienes comunes, economía participativa), el derecho (derecho constitucional, derechos humanos) y la ciencia política (democracia radical, empoderamiento-emancipación) nos disponemos a encontrar ahora los ingredientes que codifican una nueva economía constituyente ${ }^{17}$ sobre la base de esta categoría de

17.- Por Economía Constituyente hacemos referencia a la idea de que es a partir de los Bienes Democráticos que la economía encuentra una puerta de entrada en los textos constitucionales, lo que abundaremos en el artículo dedicado a explorar la vía jurídica. 


\section{LOS “BIENES DEMOCRÁTICOS» EN LA ECONOMÍA DEL BIEN COMÚN: CUATRO VÍAS PARA SU DELIMITACIÓN CONCEPTUAL}

bienes, como elemento central de la Economía del Bien Común. Cuatro vías en paralelo nos confirman el reconocimiento del conjunto de los Bienes Democráticos dando apoyo y soporte a nuestras pretensiones. Su exposición cerrará este trabajo introductorio, dando lugar a cada una de las vías y los correspondientes desarrollos que trataremos de elaborar con posterioridad.

1. Vía socio-histórica. En su aparición a lo largo de la evolución humana, estos bienes pueden verse como hitos que marcan las grandes discontinuidades o transiciones.

2. Vía económica. El trabajo efectuado por Ostrom (1990), Nobel en 2009 , consolida la categoría de propiedad y el modo de gestión de los bienes comunes como eficaz y solvente.

3. Vía política. La nueva democracia o democracia radical nos servirá para explicar cómo pueden las comunidades participar en decisiones comunitarias.

4. Vía jurídica. Los DDHH y el Derecho Internacional nos conducen a una primera aproximación del conjunto de sectores que ha de formar parte de los Bienes Democráticos.

\section{La vía socio-histórica}

Si los Bienes Democráticos son realmente factores de importancia clave para la vida humana, la aparición de cada uno de ellos debería poder asociarse a momentos verdaderamente significativos del desarrollo de las civilizaciones. En un artículo que se encuentra en fase de preparación, nos proponemos rastrear la Historia para encontrarlos y poner en valor el papel que jugaron en el proceso evolutivo de la especie humana. La primera etapa del recorrido humano comienza hace varios millones de años, con los primeros humanos siguiendo un esquema de vida básicamente idéntico al del resto de las especies del reino animal: recolectar y cazar especies vegetales y animales para alimentarse. Esta forma de vida representa el $99 \%$ de la historia de las comunidades humanas y las lleva a recorrer grandes distancias en busca de alimento -nomadismo paleolítico. Pero hace varios miles de años se produjo la primera gran discontinuidad de la Historia. El descubrimiento de las leyes que gobiernan los ciclos vitales de la naturaleza y la presión por el aumento demográfico, desembocaron en sistemas de agricultura y ganadería -el Neolítico. La agricultura les obliga a asentarse y crear ciudades permanentes y estables, por lo que esta etapa se corresponde con la aparición de la función de infraestructura: vivienda, agro-alimento y agro-textiles. Este periodo conduce a una segunda bubestación neolítica: Mesopotamia y el Valle del Nilo, dando entrada a la Edad de los Metales, donde la aparición de los primeros sistemas de irrigación (ríos artificiales, con presas, recámaras y canales para el transporte del agua desde el río natural) intensifica la producción de comida para una población mucho más numerosa. Sin depender tan directamente del régimen de lluvias como principal condición de fertilidad, se dan "los primeros pasos hacia la creación de sociedades jerárquicas estratificadas" (Ponting 1992:89-96), con contabilidad, Estado, templo y ejército completamente estructurados y bien diferenciados. Otra prueba más de la importancia del agua como factor multiplicador de evolución es que en China este mismo proceso fuese mucho más lento, posiblemente como consecuencia del "lento desarrollo de los sistemas de riego" (Ponting 1992:95). El transporte y la energía, incluyendo su forma más versátil, la electricidad, no van a experimentar avances prácticamente hasta la segunda gran discontinuidad, es decir, la revolución industrial, que se extiende por los siglos XVIII 
y XIX, continuando con una vertiginosa expansión de estilo de vida y consecuencias durante el siglo $X X$. En todo ese tiempo, el petróleo y otros fósiles han liderado las fuentes de energía primaria. Sin la ingente cantidad de energía disponible que nos han proporcionado no podría haberse dado el contexto industrial y posterior turbo-consumo (Lipovetsky 2007:10). Una tercera gran discontinuidad está sucediendo en las primeras décadas del siglo XXI, comprendiendo una primera etapa de globalización, con la sociedad-red que describe Castells, donde aparece internet como sistema para las comunicaciones y una segunda etapa que comienza a despuntar, caracterizada por una relocalización por motivos fundamentalmente ambientales, de la que hemos hablado en este trabajo. Podemos plantear la hipótesis de si será al final de esta tercera gran discontinuidad cuando los sistemas educativo y sanitario experimenten una enorme expansión, precisamente como consecuencia de una civilización basada en la confianza y unos intercambios económicos sobre la base de un principio de suficiencia (renta básica). Hay que destacar que la primera discontinuidad llevó varios milenios, la segunda varios siglos y si la serie matemática se confirma, la tercera, actualmente en tránsito, podría completarse en cuestión de décadas, algo perfectamente compatible con la aceleración del desarrollo que estamos percibiendo en nuestros tiempos. ${ }^{18}$ Junto al Bien Democrático de las comunicaciones tecnológicas, en esta tercera gran transición civilizatoria están emergiendo nuevas realidades con repercusiones económicas, políticas y jurídicas que a continuación se explican.

\section{La vía económica}

En un próximo artículo abordaremos las bases económicas que sustentan la justificación de los Bienes Democráticos de la EBC, como las necesidades humanas básicas -con mayor grado de detalle- y los bienes comunes, que aportan el modo básico de gestión para unos máximos de democracia corporativa, a partir del pensamiento y los desarrollos explorados por Ostrom (1990). Además de los bienes democráticos, anidaremos otras tres subcategorías dentro de una nueva clasificación para los bienes comunes: los bienes naturales (o ambientales), los bienes neutros y los bienes públicos. Estableceremos entre todos ellos elementos de especificación y diferenciación. Este sistema de categorías reaparecerá de nuevo para la creación de un diseño que permita distinguir entre diferentes grados de uso o consumo de los bienes democráticos (lo que hemos llamado tramo) de tal modo que, por poner un ejemplo, el agua que ha de satisfacer la necesidad básica de cualquier ser humano no se confunda administrativamente con la utilizada para satisfacer una demanda de lujo, como pueda ser la de regar un campo de golf. Nuestro recorrido partirá del contexto de la economía civil de Bruni y Zamagni (2007), con quienes exploraremos, alternando momentos de coincidencia con otros de alejamiento, el principio de reciprocidad que genera bienes relacionales y que nos encamina hacia una nueva concepción, la reciprocidad holística o sistémica.

18.- Esta aceleración es observada y formulada como teorema por Leslie White: "a medida que evoluciona la cultura, el régimen de crecimiento es acelerado" (White, 1949: 452). 


\section{LOS “BIENES DEMOCRÁTICOS» EN LA ECONOMÍA DEL BIEN COMÚN: CUATRO VÍAS PARA SU DELIMITACIÓN CONCEPTUAL}

\section{La vía Política}

La tercera gran transición de las comunidades humanas ha podido comenzar después de que la democracia de las comunicaciones (internet) haya abierto campo para desarrollos paralelos en la economía (colaborativa) y en política (democracia participativa y directa) hasta el punto de que en los próximos años se esperen cambios en estos ámbitos tan fabulosos como el fin de la era del empleo que pronostica Rifkin (1996). La sociedad-red de Castells (1998) que emerge de la globalización ha dejado paso a una relocalización, como segunda fase de un mismo proceso que puede desembocar en una ruptura que renueve el contrato social (Rousseau 1762), una vez probada la insuficiencia de un orden basado puramente en empleos escasos, infra-remunerados o carentes de sentido y que debe encontrar una nueva base -capaz de aunar necesidades ambientales, emocionales y materiales- sobre la que edificar el nuevo contrato. Explicaremos esta vía en otro artículo, en el que un ordenamiento básicamente distinto a lo que mercado y Estado por sí mismos pueden proponer, empieza a asomar. Con la incorporación de la sociedad civil, su fraternidad, su soberanía, su emancipación y su gobernanza, las bases de ese nuevo modelo parecen encontrarse en el tejado del procomún, en la zona que Hardin trató de dejar cerrada para siempre en 1968, pero que una mujer, la primera y única ganadora hasta hoy del Nobel de economía (2009), ha vuelto a abrir, después de demostrar la inconsistencia del prejuicio neoliberal acerca de la manera idónea con que puede gestionarse un bien.

\section{La vía jurídica}

¿Son todos los bienes iguales? Y en caso de respuesta afirmativa, ¿por qué aparece entonces la vivienda como derecho constituyente en solitario? La Constitución representa, por encima de cualquier otra consideración, una separación jurídica entre la norma fundamental y la ordinaria, por el efecto institucionalizante que ejerce la propia separación que asigna a cuanto queda incluido en ella, de inmediato investido (en realidad, legitimado) como fundamental, respecto de cualquier otro derecho, que queda declarado como (en realidad, reducido a) ordinario. Esta función que ejerce, convierte a la Constitución en un principio de división. ${ }^{19}$ Sin ese carácter, generador de la categoría de lo fundamental, la carta sería una mera guía de método, quedando notablemente acortada, con el único capítulo y función de ordenar la forma de poner en funcionamiento los mecanismos legítimos para la toma de decisiones, según el principio de mayoría. Por el contrario, una lista de derechos derivados del derecho internacional quedan salvaguardados como fundamentales. El concepto de Bienes Democráticos encuentra su justificación también desde el ámbito del derecho, precisamente en este mismo tipo de operación. Si trasladamos esta divisoria desde lo jurídico al ámbito de la economía, establecemos una categoría paralela por la que formalmente aparecerían bienes, que si bien no pueden calificarse de fundamentales -no se trata de principios, sino de bienes, es decir, entes que se producen, se distribuyen y se consumen- sí puede decirse de ellos que son -han de ser- democráticos, como forma equivalente a "fundamental" en la práctica de su gestión. Por lo tanto, desde el punto de vista jurídico, podemos defi-

19.- Una explicación detallada de este mismo efecto, pero aplicado al ritual puede verse en la fuerte crítica que hace Pierre Bourdieu a las teorías sobre los ritos de paso de Van Gennep y Victor Turner (1985:78-80). 
nir los Bienes Democráticos constituyentes como la extensión desde el derecho a la economía de la división que genera la categoría que declara inviolable el listado mínimo de elementos que pone en marcha una sociedad justa de personas moralmente equivalentes 0 una red que amortigua la caída al vacío en una sociedad de riesgo (Beck 1992) y que por fin comienza a gestar otro mundo con posibilidades de resiliencia. La propuesta legislativa de incluirlos equivale a establecer como derecho tanta democracia como fuere posible en aquellos sectores absolutamente indispensables para la vida y la dignidad humanas, siendo ese paquete mínimo, el formado por vivienda, agroecología, agua y energía eléctrica, acceso a internet y a la movilidad urbana, y a los sistemas educativo y sanitario. Al igual que con las vías anteriores, dejamos el grueso de este trabajo para un artículo específico posterior.

\section{Bibliografía}

ARISTÓTELES: La Política, Libro I, Colección Austral, Espasa Calpe, 1999.

AZKARRAGA, J. (2014): "Resiliencia local y comunitaria frente a la crisis sistémica". En: J.M. Madariaga (Coord.), Nuevas miradas sobre la resiliencia. Ampliando ámbitos y prácticas, Barcelona: Gedisa, 85-110.

BECK, U. (1992): Risk Society: Towards a new modernity, London: Sage.

BOURDIEU, P. (1985): ¿Qué significa hablar?, Madrid: Akal.

BRUNI, L. \& ZAMAGNI, S. (2007): Economía Civil. Eficiencia, equidad, felicidad pública, Buenos Aires: Prometeo.

CASTELLS, M. (1998): La era de la información. Economía, sociedad y cultura. Vol. 1: La sociedad red, Madrid: Alianza Editorial.

COMITÉ ECONÓMICO Y SOCIAL DE LA UNIÓN EUROPEA (2015): La Economía del Bien Común: un modelo económico sostenible orientado a la cohesión social, Dictamen ECO/378 - EESC2015-02060-00-00-AC-TRA (ES) 1/113. Ponente Carlos Trías Pintó, co-ponente Stefano Palmieri. Bruselas, 17 de septiembre de 2015.

CONSEJO EUROPEO DE COPENHAGUE, Conclusiones de la Presidencia, 21-22 de junio de 1993, SN 180/1/93 REV1.

FELBER, C. (2010): La Economía del Bien Común, Barcelona: Deusto.

FORÉS, A., GRANÉ, J. \& OLLÉ, R.M. (2014): "Generando posibilidades. Organizaciones resilientes. Organizaciones con futuro". En: J.M. Madariaga (Coord.), Nuevas miradas sobre la resiliencia. Ampliando ámbitos y prácticas, Barcelona: Gedisa, 227-248. 


\section{LOS “BIENES DEMOCRÁTICOS» EN LA ECONOMÍA DEL BIEN COMÚN: CUATRO VÍAS PARA SU DELIMITACIÓN CONCEPTUAL}

GALTUNG, J. (1977): Human Needs as the focus of Social Sciences, manuscrito gris (no publicado). Disponible en http://www.transcend.org/galtung/papers.php?q=human+needs.

GIL, G.E. (2014): "Fundamentos y aplicación de la resiliencia holística". En: J.M. Madariaga (Coord.), Nuevas miradas sobre la resiliencia. Ampliando ámbitos y prácticas, Barcelona: Gedisa, 191-207.

HARDIN, G. (1968): "The tragedy of the commons", Science, 162, 1243-1248.

HELD, D. (2006): Global Covenant. The Social Democratic Alternative to the Washington Consensus, Reino Unido: Polity.

LIPOVETSKY, G. (2007): La felicidad paradójica. Ensayo sobre la sociedad de hiperconsumo, Barcelona: Anagrama.

MADARIAGA, J.Mª (2014): "La construcción social de la resiliencia". En: J.M. Madariaga (Coord.), Nuevas miradas sobre la resiliencia. Ampliando ámbitos y prácticas, Barcelona: Gedisa, 11-30.

ORGANIZACIÓN DE NACIONES UNIDAS (1948): Declaración Universal de Derechos Humanos, 13 de diciembre de 1948, Naciones Unidas, [en linea] http://www.un.org/es/documents/udhr.

OSTROM, E. (1990): Governing the commons: The evolution of Institutions for Collective Action, Cambridge: University Press.

PONTING, C. (1991): Historia verde del mundo, Barcelona: Paidós.

REICH, J.W., ZAUTRA, A.J. \& HALL, J.S. (2010): Handbook of Adult Resilience, Nueva York: The Guilford Press.

RIFKIN, J. (1995): El fin del trabajo. Nuevas tecnologías contra puestos de trabajo: el nacimiento de una nueva era, Barcelona: Paidós.

ROUSSEAU, J.J. (1762): El contrato social. Los principios del derecho político, Maxtor, ed. 2008.

SUÁREZ OJEDA, N. et al. (2001): Resiliencia. Descubriendo las propias fortalezas. Buenos Aires: Paidós.

UE (1992): Tratado de la Unión Europea, de 7 de febrero de 1992 firmado en Maastricht, DOUEC, № 340, de 10 de noviembre de 1997 y BOE de 13 de enero de 1994.

WHITE, L. (1949): La ciencia de la cultura. Un estudio sobre el hombre y la civilización, Círculo de lectores, ed. 1988.

WRIGHT, E.O. (2015): "How to be an anticapitalist today". Transcend Media Service. 14 de diciembre de 2015. Jacobin Magazine. [en linea]. <https://www. transcend.org/tms/?p=67437>. [consulta: 16 de diciembre de 2015]. 


\section{Anexo}

\section{Los Bienes Democráticos. Funciones y Necesidades Básicas asociadas}

\begin{tabular}{|l|ll|l|}
\hline Función & Bienes democráticos & Necesidad básica \\
\hline Reproducción & Sanidad & Educación & Identidad \\
Comunicación & Internet & Movilidad & Libertad \\
Suministro & Electricidad & Agua & Bienestar \\
Infraestructura & Vivienda & Agroecología & Supervivencia \\
\hline
\end{tabular}

